PHYSICAL REVIEW D 96, 049903(E) (2017)

\title{
Publisher's Note: First law and anisotropic Cardy formula for three-dimensional Lifshitz black holes [Phys. Rev. D 91, 064006 (2015)]
}

Eloy Ayón-Beato, Moisés Bravo-Gaete, Francisco Correa, Mokhtar Hassaïne, Maria Montserrat Juárez-Aubry, and Julio Oliva (Received 25 July 2017; published 7 August 2017)

DOI: 10.1103/PhysRevD.96.049903

This paper was published online on 2 March 2015 with errors in the author affiliations and indicators. The author list and affiliation indicators should read as

Eloy Ayón-Beato, ${ }^{1,2 *}$ Moisés Bravo-Gaete, ${ }^{3, \dagger}$ Francisco Correa, ${ }^{4,5}$ Mokhtar Hassaïne, ${ }^{3, \S}$ María Montserrat JuárezAubry, ${ }^{1,2,6, \|}$ and Julio Oliva ${ }^{2, \pi}$

${ }^{1}$ Departamento de Física, CINVESTAV-IPN, Apartado Postal 14-740, 07000 Mexico D.F., México

${ }^{2}$ Instituto de Ciencias Físicas y Matemáticas, Universidad Austral de Chile, Casilla 567, Valdivia, Chile

${ }^{3}$ Instituto de Matemática y Física, Universidad de Talca, Casilla 747, Talca, Chile

${ }^{4}$ Leibniz Universität Hannover, Appelstraße 2, 30167 Hannover, Germany

${ }^{5}$ Centro de Estudios Cientficos (CECs), Casilla 1468, Valdivia, Chile

${ }^{6}$ Instituto Tecnológico y de Estudios Superiores de Monterrey, Campus Puebla, Va Atlixcáyotl No. 2301, Reserva Territorial Atlixcáyotl, Puebla, C.P. 72453 Puebla, México

The author affiliations and indicators have been corrected as of 2 August 2017. The author affiliations and indicators are incorrect in the printed version of the journal. 